\title{
Are we familiar with this rare indicative of a higher risk for breast cancer?
}

\author{
Ayşegül Altunkeser ${ }^{1} \odot$, Fatma Zeynep Arslan$^{1} \odot$, Ender Alkan²$^{2}$, Tuğba Günler $^{3} \odot$ \\ ${ }^{1}$ Department of Radiology, University of Health Sciences, Konya Training and Research Hospital, Konya, Turkey \\ ${ }^{2}$ Department of Radiology, Aksaray University, Aksaray Training and Research Hospital, Aksaray, Turkey \\ ${ }^{3}$ Department of Pathology, University of Health Sciences, Konya Training and Research Hospital, Konya, Turkey
}

DOI: $10.18621 /$ eurj.424563

\begin{abstract}
Secretory adenosis (SA) of breast is rarely seen benign breast lesion, which might be associated with increased risk for breast carcinoma. SA is an extremely rare lesion, the cases reported in the literature and long-term follow-up studies are limited and radiological and histopathological diagnosis of SA is mostly challenging; it could be frequently misinterpreted as ductal carcinoma in situ. Because of these reasons; clinical significance and management of SA is still not fully understand and relative risk of SA is still not well-established. Herein; we presented mammography, ultrasound, magnetic resonance imaging and microscopic findings in a patient with SA of breast.
\end{abstract}

Keywords: breast carcinoma, magnetic resonance imaging, secretory adenosis

Received: May 17, 2018; Accepted: August 3, 2018; Published Online: January 18, 2019

$\mathbf{S}$ ecretory adenosis (SA) of the breast is rarely seen type of sclera osing lesions [1, 2]. There is a significant increase in proliferation in both glandular epithelium and myoepithelial $[3,4]$. There are only a few recent researches investigating the frequency of SA; prevalence of SA is reported as approximately $0.4 \%$ [5]. SA is an extremely rare lesion, the cases reported in the literature and long-term followup studies are limited and radiological and histopathological diagnosis of SA is mostly challenging; especially for SA with or without atypical could be frequently misinterpreted as ductal carcinoma in situ [6]. Because of these reasons; clinical significance and management of SA is still not fully understand and relative risk of SA is still not well-established. Considering the uncommon nature of SA and importance of reporting such unique lesions; we presented here a mammography, ultrasound, magnetic resonance imaging (MRI) and microscopic findings.

\section{CASE PRESENTATION}

A 44-year-old female admitted to our outpatient clinic with a palpable mass on the right breast. There was no known medical history and laboratory findings were normal. On physical examination; a palpable, soft and mobile mass was detected in outer quadrant of right breast. On mammography; encapsulated heterogeneous nodular density containing lucent areas in a size with $40 \times 20 \mathrm{~mm}$ was observed (Figure 1). Sonographically; a well-circumscribed, heterogeneous hyperechoic lesion in a size with $40 \times 20 \mathrm{~mm}$ was seen. Microcalcifications were observed within the

Address for correspondence: Fatma Zeynep Arslan, MD., University of Health Sciences, Konya Training and Research Hospital, Department of Radiology, Konya, Turkey

E-mail: Zzynep_a1002@hotmail.com,Fax:+903323236723 

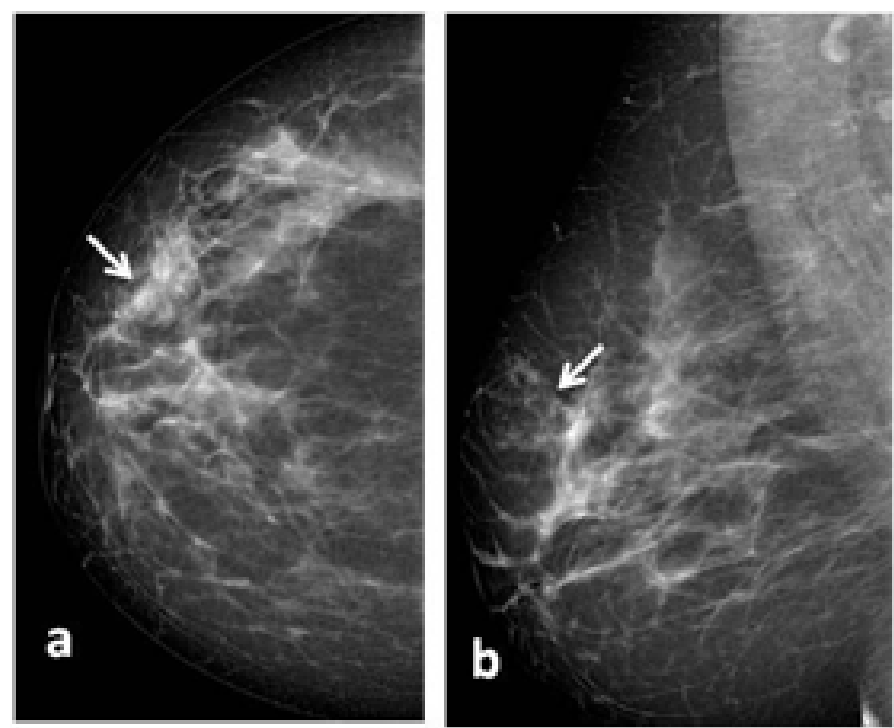

Figure 1. Craniocaudal (a) and mediolateral oblique (b) mammogram of the right breast has demonstrated an encapsulated heterogeneous nodular density containing lucent areas (arrow).

lesion. MRI demonstrated encapsulated nodular lesion of approximately $3 \mathrm{~cm}$ in diameter with early phase contrast enhancement and type 2 contrast enhancement pattern was seen in the late phase. Diffusion restriction was detected on diffusionweighted imaging (Figure 2). Clinical and radiological data could not exclude malignancy, a core biopsy was taken with 16 Gauge needle. On histopathological examination, ductal structures are observed between the layer of adipose tissue, hyalinized stroma, and myoepithelial layers. Secretion was noted in the lumen of someducts. In the immunohistochemical study performed, positive staining for calponin was seen (Figure 3). Lesion is confirmed as secretory adenosis with marked cystic degeneration.

\section{DISCUSSION}

Secretary adenosis is mostly occurred in the salivary gland, skin, and parathyroid, breast is an unusual location [7]. SA affects both young and old women. SA can be seen with fibrocystic changes or can be observed as a pure entity [8]. While some important studies $[5,9]$ reported that SA is a benign, some studies found that there is a RR increase for developing future malignancy [10]. A research with a larger sample size found that there is an important relative risk increase about 5 times for future breast malignancy [10]. There is also some evidence in the literature that an excised SA may recur [11]. Literature
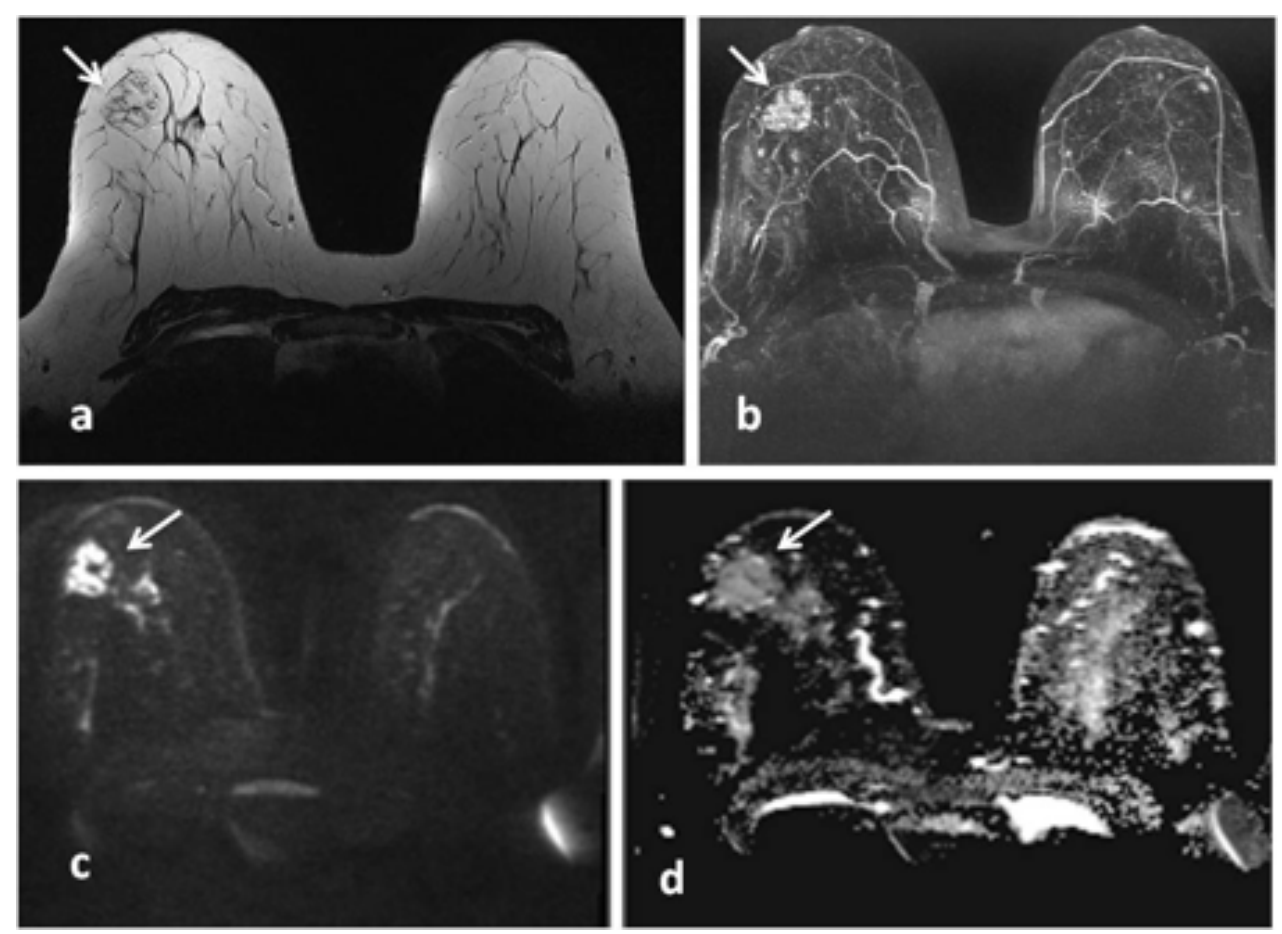

Figure 2. Axial T2 weighted image demonstrates an encapsulated mass (a), MIP imagehaverevealed a masswithtype 1 contrastenhancementpattern (b), DWI (c), and ADC mapping (d) demonstrates diffusion restriction of mass (arrow). MIP = maximum intensity projection, DWI = diffusion weighted imaging, $\mathrm{ADC}=$ apparent diffusion coefficient. 

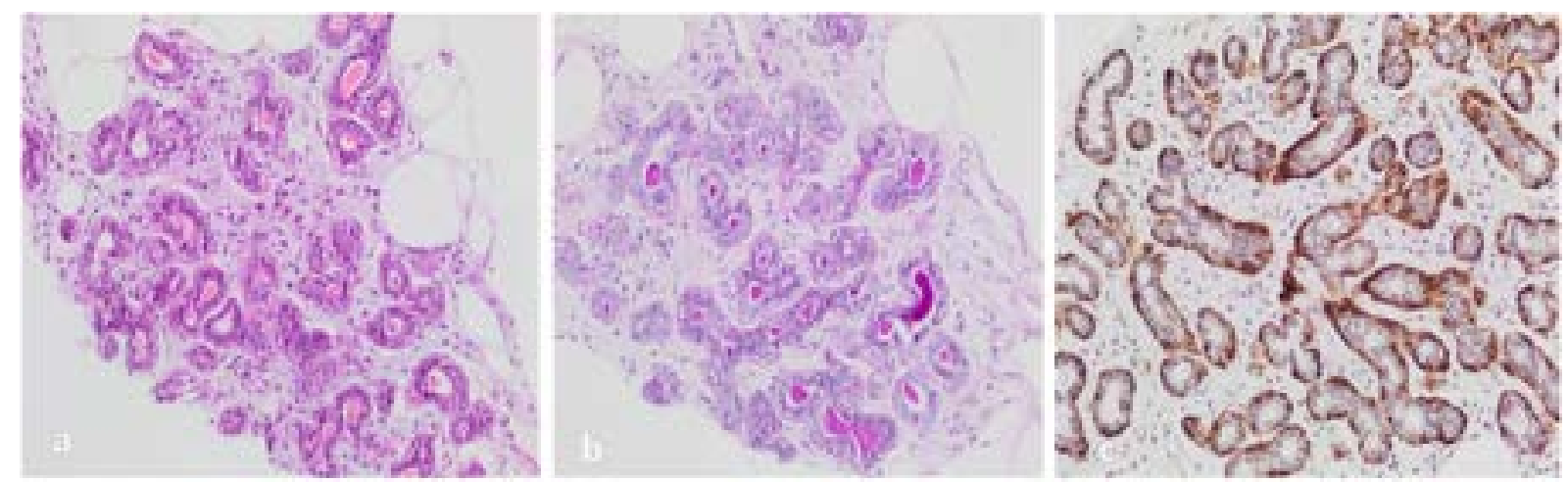

Figure 3. On Periodic acid-Schiff positive (a), diastase-resistant (b) sections, secretion is noted in small glands within traluminal eosinophilic secretions. Myoepithelial cells that are positively stained with the calponin around the secretory glands are seen (c).

data has revealed such various results; since lesion is rarely encountered and there are only few studies and case reports in the literature.

On physical examination a lump can be palpable in the breast, on mammography asymmetric focal density or mass lesion with diffuse or clustered microcalcifications with an irregular shape or spiculated borders, or display asymmetric focal density and focal architectural distortion may be seen, which could support the suspicion of breast cancer [12]. We also observed an encapsulated focal asymmetric density containing radiolucent areas on mammography. The mammographic appearance of lesion was similar to hamartoma. SA revealed that hypoechoic mass with a uncertain boundary or well circumscribed mass with microlobulated contours can be sonographically seen $[12,13]$. We observed a wellcircumscribed, heterogeneous hyperechoic lesion containing microcalcifications on ultrasound. Yet, no sonographically specific feature is described. It seems almost impossible to obtain accurate diagnosis based on mammographic and sonographic findings. Gity et al. [14] emphasized MRI as a usefull modality in characterizing lesions containing pure adenosis from mixed adenosis lesions. They also revealed that adenosis lesions are frequently false-positively reported as malign, since these lesions can show morphologic or dynamic border line features on MRI. In a vast majority of these adenosis showed oval/lobulated or irregular shapes, showed heterogeneous internal enhancement, all demonstrated a rapid initial rise then wash-out enhancement patterns on dynamic examination with varying enhancement patterns such as seg-mental or linear distribution and clumped internal enhancement [14]. In our patient; we detected a nodular lesion with an contrast enhancement in early phase and type 2 contrast enhancement pattern was seen in the late phase on dynamic MRI, too. A core needle biopsy and histopathological confirmation is necessary for the definitive diagnosis. On microscopic examination; haphazard proliferation of bland glands with apocrine differentiation with apocrine metaplasia in $>50 \%$ of adenosis area is seen [15]. On microscopic examination of our case, myoephitelial cells were seen. Treatment options include follow-up or excision of the lesion. The differential diagnosis also includes sclerosing adenosis, fibroadenoma, ductal carcinoma in situ and breast carcinoma.

\section{CONCLUSION}

In conclusion; SA is rare and there is only limited number of cases have been reported. Relative risk of developing possible future malignancy and interval to cancer diagnosis as mean time is yet not fully established. It is a great necessity to report these rare SA cases and to perform new researches could provide important informations for understanding SA.

\section{Informed consent}

Written informed consent was obtained from the patient for publication of this case report and any accompanying images. 


\section{Conflict of interest}

The authors declared that there are no potential conflicts of interest with respect to the research, authorship, and/or publication of this article.

\section{REFERENCES}

[1] Tavassoli FA, Soares J. Myoepithelial Lesion: World Health Organization classification of Tumors. Pathology\& Genetics. Tumors of the breast and Female Genital Organs. Lyon: International Agency for Research on Cancer (IARC); 2003:868.

[2] Moinfar F. Adenosis. In Essentials of Diagnostic Breast Pathology. Edited by: Moinfar F. Berlin: Springer; 2007:31.

[3] Tsuda H, Mukai K, Fukutomi T, Hirohashi S. Malignant progression of adenomyoepithelial adenosis of the breast. Pathol Int 1994;44:475-9.

[4] Tavassoli FA. Myoepithelial lesion of the breast. Myoepitheliosis, adenomyoepithelioma, and myoepithelial carcinoma. Am J Surg Pathol 1991;15:554-68.

[5] Page DL, Dupont WD, Jensen RA. Papillary apocrine change of the breast: associations with atypical hyperplasia and risk of breast cancer. Cancer Epidemiol Biomarkers Prev 1996;5:29-32. [6] Watanabe K, Nomura M, Hashimoto Y, Hanzawa M, Hoshi T. Fine needle aspiration cytology of apocrine adenosis of the breast: Report on three cases. Diagn Cytopathol 2007;35:296-9.
[7] Mitra B, Pal M, Saha TN, Maiti A. Adenomyoepithelial adenosis of breast: a rare case report. Turk Patoloji Derg 2017;33:240-3.

[8] Rosen PP. Rosen's Breast Pathology. 3rd ed. Philadelphia: Lippincott Williams \&Wilkins; 2008.

[9] Calhoun BC, Booth NC. Atypical apocrine adenosis diagnosed on core biopsy: implications for management. Hum Pathol 2014;45:2130-5.

[10] Seidman JD, Ashton M, Lefkowitz M. Atypical apocrine adenosis of the breast: a clinico pathologic study of 37 patients with 8.7-year follow-up. Cancer 1996;77:2529-37.

[11] Erel S, Tuncbilek I, Kismet K, Kilicoglu B, Ozer E, Akkus MA. Ademomyoepithelial adenosis of the breast: clinical, radiological, and pathological findings for diffrential diagnosis. Breast Care 2008;3:427-30.

[12] Günhan-Bilgen I, Memiş A, Ustün EE, Ozdemir N, Erhan Y. Sclerosing adenosis: mammographic and ultrasonographic findings with clinical and histopathological correlation. Eur J Radiol 2002;44:232-8.

[13] Chen JH, Nalcioglu O, Su MY. Fibrocystic change of the breast presenting as a focal lesion mimicking breast cancer in MR imaging. J Magn Reson Imaging 2008;28:1499-505.

[14] Gity M, Arabkheradmand A, Taheri E, Shakiba M, Khademi Y, Bijan B, et al. Magnetic resonance imaging features of adenosis in the breast. J Breast Cancer 2015;18:187-94.

[15] Gillellamudi SB, Vellanki VS, Veeragandham S, Omar O. Apocrine adenosis of breast: a very rare case report. Int Surg J 2016;1:97-8. 